\title{
Laser-Induced Resonance Shifts of Single Molecules Self-Coupled by a Metallic Surface
}

\author{
O. Labeau, ${ }^{1}$ Ph. Tamarat, ${ }^{1}$ H. Courtois,${ }^{2}$ G. S. Agarwal,${ }^{3}$ and B. Lounis ${ }^{1, *}$ \\ ${ }^{1}$ Centre de Physique Moléculaire Optique et Hertzienne, UMR 5798 CNRS et Université Bordeaux 1, \\ 351 Cours de la Libération, 33405 Talence, France \\ ${ }^{2}$ Institut Néel, CNRS and Université Joseph Fourier, 25 Avenue des Martyrs, 38042 Grenoble, France \\ ${ }^{3}$ Department of Physics, Oklahoma State University, Stillwater, Oklahoma 74078, USA
}

(Received 9 October 2006; published 5 April 2007)

\begin{abstract}
The spectral properties of single molecules placed near a metallic surface are investigated at low temperatures. Because of the high quality factor of the optical resonance, a laser-induced shift of the molecular lines is evidenced for the first time. The shift dependence on the laser excitation intensity and on the dephasing rate of the transition dipole is studied. A simple theoretical model of a laser-driven molecule self-coupled by a mirror is developed to qualitatively interpret the observations.
\end{abstract}

PACS numbers: 33.80.- b, 32.70.Jz, 33.50.- j, 42.50.-p

The radiative properties of an oscillating electric dipole are influenced by the boundary conditions [1,2]. For example, when a dipole is placed within a few wavelengths of a conducting surface, its decay rate and resonance frequency are changed from those observed for an isolated dipole. There have been many theoretical works on the calculation and interpretation of these phenomena using classical or quantum electrodynamics approaches [3-8]. Since the pioneering work of Drexhage [9], who focused on the dependence of the fluorescence lifetime of $\mathrm{Eu}^{3+}$ ions on their distance to a metallic surface, extensive experimental investigations of this effect have been reported with various systems and in various geometrical configurations [1]. Some experimental observations of the energy level shifts of atoms interacting with a reflecting surface have also been reported in the cases of an atomic vapor close to a metallic film [10], and a single trapped ion distant from a mirror [11].

Single fluorescent molecules in solids [12] are good candidates to investigate these effects. Recently, the fluorescence lifetime and intensity of a single molecule at room temperature have been studied as a function of its distance to a movable silver mirror [13], and to a metallic nanoparticle at the end of a tip [14,15]. At low temperatures $(<2 \mathrm{~K})$, dephasing of the transition dipole due to phonons vanishes, and for well chosen fluorophore-matrix systems the zero phonon excitation line has a spectral width limited by the excited state lifetime [16]. Such molecules behave like two-level systems [17] with a fluorescence quantum yield close to unity, thus offering optical properties similar to those of trapped single atoms. In this work, we exploit the high quality factor of this resonance to investigate the spectral properties of laser-driven single molecules close to a metallic surface. A spectral shift of the resonance induced by the excitation laser is evidenced for the first time and is studied under various experimental conditions. A simple theoretical model based on optical Bloch equations of an oscillating dipole interacting with its mirror image is developed. It allows us to make the link between the observed laser-induced shifts (LIS) and the surface modified energy levels of the molecule.

In this experiment, we study the fluorescence excitation spectra of a thin organic film doped with fluorescent molecules and deposited on a metallic layer prepared by evaporation. Scans of the zero phonon line of single molecules are recorded using a single frequency tunable dye laser. The sample, cooled in a helium flow cryostat, is placed at the focal plane of a parabolic mirror used in a confocal setup to excite and collect the fluorescence of the molecules [18]. The organic layer thickness is estimated by stoichiometry, i.e., counting the number of single excitation lines recorded when the laser frequency is scanned over the entire inhomogeneous absorption band, for a known fluorophore concentration and a known laser spot size. All the results presented hereafter have been obtained with organic layers of thickness $<1 \mu \mathrm{m}$.

Most of the single molecule excitation lines recorded at very low excitation intensities display a width close to its value in the absence of metal layer. For dibenzanthanthrene (DBATT) fluorophores in a hexadecane matrix at $1.7 \mathrm{~K}$ [19], this metal-unaffected lifetime-limited width $\Gamma_{0}$ takes the value $\Gamma_{0} / 2 \pi \approx 17 \mathrm{MHz}$. However, in some cases (below $5 \%$ in the thinnest samples), spectra reveal very broad single molecule lines, as illustrated in Fig. 1. This broadening is not due to optical saturation and is attributed to a quenching effect corresponding to nonradiative energy transfer to the metal when the molecules are within $10 \mathrm{~nm}$ from the surface $[6,13]$. To our knowledge, this is the first experimental observation of a single molecule line broadening due to quenching by a metal surface.

A more striking effect evidenced in our experiment is a reversible spectral shift displayed by some molecular lines when the laser intensity is increased. Figure 2(a) shows such a behavior for a single DBATT molecule close to a niobium film at $1.7 \mathrm{~K}$. The LIS is clearly visible for single molecules with sharp excitation lines (linewidths close to $\left.\Gamma_{0}\right)$, i.e., molecules which are several tens of nanometers away from the metal surface $[6,13]$. The optical saturation 


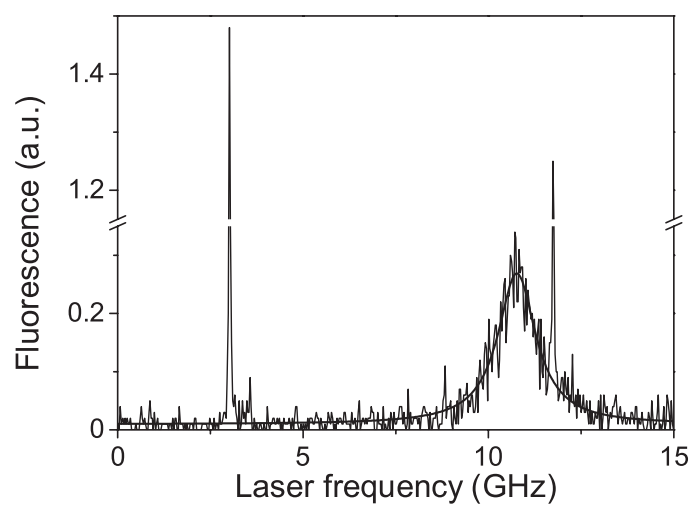

FIG. 1. Fluorescence excitation spectrum at $T=1.7 \mathrm{~K}$ for three single DBATT molecules embedded in a hexadecane layer deposited on a $50 \mathrm{~nm}$ thick niobium substrate. The spectrum is recorded at an excitation intensity well below saturation. Two sharp lines display a nearly surface-unaffected linewidth $(\sim 20 \mathrm{MHz})$. The broad Lorentzian line has a linewidth of $1.1 \mathrm{GHz}$.

of the molecules follows the same behavior as in the case of samples deposited on dielectric substrates [19]. Figure 2(b) shows the linewidth intensity dependence of the molecule presented in Fig. 2(a). It evolves as $\Delta \nu_{\text {hom }} \sqrt{1+I / I_{\text {sat }}}$, where $I / I_{\text {sat }}$ is the ratio of the laser excitation intensity to the molecule saturation intensity. At this low temperature, the homogeneous width is determined by the excited state decay rate $\Gamma$. Deduced from the fit [see Fig. 2(b)], $\Delta \nu_{\text {hom }}=\Gamma / 2 \pi \approx 18 \mathrm{MHz}$ is close to the metal quenching-free value. The spectral shift of the resonance shows a linear dependence on the excitation intensity up to large saturations with a slope of $1.2 \mathrm{MHz} /\left(\mathrm{W} \mathrm{cm}^{-2}\right)$ [see Fig. 2(c)], i.e., $0.11 \Delta \nu_{\text {hom }}$ per saturation intensity.

The LIS, which is completely absent with dielectric substrates, is found for various metal layers (silver, aluminum, gold, indium, niobium) of different thicknesses (from a few nanometers to hundreds of nanometers). For all the metallic films studied, the roughness of the metallic surface was controlled by atomic force microscopy and was about $1 \mathrm{~nm} \mathrm{rms}$, excluding electromagnetic field enhancement effects. Moreover, the behavior was found for different host-guest systems: different fluorophores (DBATT, terrylene, and tetra-tert-butylterrylene) in different matrices (naphthalene or $p$-terphenyle molecular crystals, and hexadecane Shpoll'skii polycrystal). For the hundred molecules investigated, red and blue spectral shifts are found with comparable probabilities and the intensity broadening was always reproduced with the optical saturation law.

In order to rule out local heating effects due to the absorption by the metal layer, we studied the LIS dependence on the orientation of the laser linear polarization, for a fixed excitation intensity, and obtained the well-known law of Malus. As shown in Fig. 2(d), the LIS is proportional to $\cos ^{2}(\theta)$ where $\theta$ is the relative angle between the
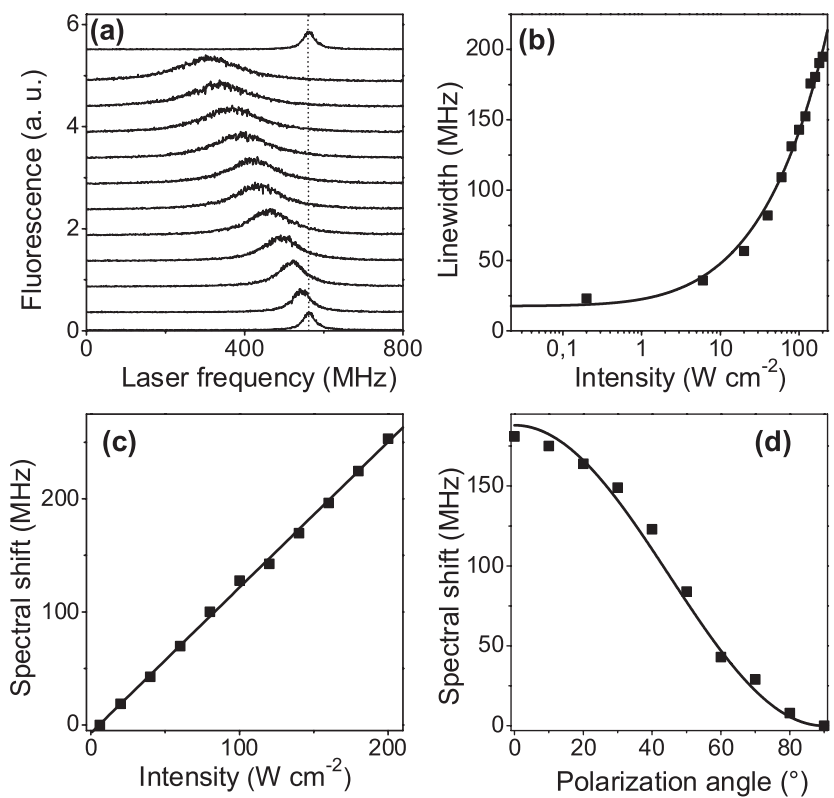

FIG. 2. LIS of a single molecule of DBATT in hexadecane near a $50 \mathrm{~nm}$ indium layer at $1.7 \mathrm{~K}$. (a) Fluorescence excitation spectra recorded at increasing excitation intensities from bottom to top (steps of $20 \mathrm{~W} \mathrm{~cm}^{-2}$ ). The top spectrum is recorded after the series at the same intensity as the bottom spectrum $\left(6 \mathrm{~W} \mathrm{~cm}^{-2}\right)$. (b) Line broadening with excitation intensity. A fit by a two-level system optical saturation law gives $\Delta \nu_{\text {hom }}=$ $18 \mathrm{MHz}$ and $I_{\text {sat }}=1.6 \mathrm{~W} \mathrm{~cm}^{-2}$. (c) Dependence of the LIS on the excitation intensity. The LIS slope is $1.3 \mathrm{MHz} /\left(\mathrm{W} \mathrm{cm}^{-2}\right)$, i.e., $0.11 \Delta \nu_{\text {hom }}$ per saturation intensity. (d) LIS dependence on the laser linear polarization which is rotated in a plane parallel to the metallic layer, at a fixed excitation intensity of $120 \mathrm{~W} \mathrm{~cm}^{-2}$. The dependence is well fitted with $\cos ^{2} \theta, \theta$ being the angle between the laser field and the transition dipole projection onto the metal surface plane.

linear polarization of the laser, which is contained in a plane parallel to the metal surface, and the projection of the transition dipole onto this plane. Since the metal absorption should not depend on the laser polarization, a contribution of metal heating to the origin of the LIS can thus be excluded. We also investigated how dephasing of the transition dipole affects the LIS by varying the temperature of the sample. The coherence decay rate $\gamma$ of the optical transition writes as $\gamma=\pi \Delta \nu_{\text {hom }}=\Gamma / 2+\gamma^{*}$, where $\Gamma$ is the excited state relaxation rate, and $\gamma^{*}$ the pure dephasing contribution due to coupling with the phonon bath. Besides the thermally activated homogeneous broadening [16], the temperature increase leads to a decrease of the LIS slope. As shown in Fig. 3 for a temperature variation between 1.8 and $4.2 \mathrm{~K}$, the slope is inversely proportional to the homogeneous linewidth. Since the saturation intensity is proportional to the coherence decay rate, the experimental results are consistent with a LIS being proportional to $I / I_{\text {sat }}$.

To understand our experimental observations, we develop a simple model to account for the environment in 


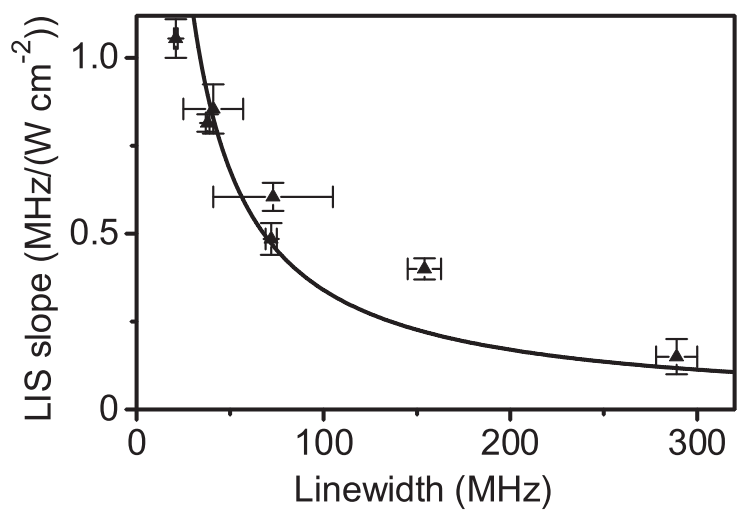

FIG. 3. Effect of transition dipole dephasing on the LIS for a single DBATT molecule in hexadecane near a $50 \mathrm{~nm}$ gold layer. The LIS slope is measured for different temperatures, varied between 1.8 and $4.2 \mathrm{~K}$, and plotted as a function of the temperature dependent homogeneous width. The experimental points are fitted by a function inversely proportional to homogeneous width.

which the molecule is radiating. We assume a two-level molecule with a transition dipole $\vec{d}_{e g}$, located at a small distance from the surface so that its interaction with the metal can be considered in the static limit. The fluorescence signal of the molecules is given by the excited state population. This population is determined from the solution of Bloch equations with the radiative decay constant $\Gamma_{0}$ replaced by the surface modified decay rate $\Gamma$, and with the laser field replaced by the local field $\vec{E}_{\text {loc }}$. This field is evaluated by taking into account the dipole-dipole interaction with the image molecule. It is the sum of the laser field, and the field arising from the image dipole which is proportional to the molecule stationary optical coherence $\rho_{e g}=-\Omega(\delta-i \gamma) /\left(\delta^{2}+\gamma^{2}+\gamma \Gamma^{-1}|\Omega|^{2}\right)$. The complex Rabi frequency $\Omega=-\left(\vec{d}_{e g} \cdot \vec{E}_{\text {loc }}\right) / \hbar$ of the molecule-local field system therefore writes as

$$
\Omega=\Omega_{L}+\frac{\beta}{2} \Gamma \frac{-\delta+i \gamma}{\delta^{2}+\gamma^{2}+\frac{\gamma}{\Gamma}|\Omega|^{2}} \Omega,
$$

where $\Omega_{L}$ and $\delta$ are, respectively, the complex Rabi frequency and the laser detuning. $\beta$ is a geometric factor which depends on the optical properties of the metallic layer, as well as on the exact location and orientation of the molecule with respect to the surface. It can be calculated using classical or quantum electrodynamics models [3$6,8]$ and lies in the range of 1-10 for a molecule-surface distance of about $100 \mathrm{~nm}$. After a simple algebraic manipulation, we obtain a transcendental equation for the excited state population $\rho_{e e}=\left(\gamma|\Omega|^{2} / 2 \Gamma\right) /\left(\delta^{2}+\gamma^{2}+\right.$ $\left.\gamma \Gamma^{-1}|\Omega|^{2}\right)$ :

$$
\rho_{e e}=\frac{1}{2} \frac{\gamma\left|\Omega_{L}\right|^{2} / \Gamma}{\left[\delta+\beta\left(\frac{1}{2}-\rho_{e e}\right) \Gamma\right]^{2}+\gamma^{2}+\gamma\left|\Omega_{L}\right|^{2} / \Gamma},
$$

which can be solved perturbatively or numerically. The effect of the metallic surface clearly shows up in Eq. (2). For very weak excitation intensities, one can set $\rho_{e e} \approx 0$ in the denominator of Eq. (2) and retrieve the self-radiation reaction shift $-\beta \Gamma / 2$ induced by the presence of the boundary conditions. In the low saturation regime where $\rho_{e e} \propto\left|\Omega_{L}\right|^{2} / \gamma \Gamma$, Eq. (2) predicts a LIS proportional to $I / I_{\text {sat }}$, with a direction depending on the sign of $\beta$. This is in broad agreement with our experimental observations. However, at high saturations the model predicts a saturation of the LIS.

Another interesting behavior has been found in the case of a gold surface. For this metal very large LIS slopes are observed (see Fig. 4), as well as a reversal of the shift direction when the system is pushed into intense coupling conditions $\left(I / I_{\mathrm{sat}} \sim 100\right)$. The surface plasmon resonance, which is close to the molecular transition, is probably responsible for the large LIS effect [20,21]. Furthermore, one would have to take into account the long-range interactions with neighboring molecules assisted by nonradiative excitation of surface plasmons [22,23]. This interaction, which can be significant at laser powers such that the LIS makes the molecule under consideration almost resonant with a neighboring one, may be at the origin of the shift direction reversal. The calculation of the local field would therefore require a detailed microscopic model accounting for the interactions between the molecule of interest and the neighboring molecules, including their images [24].

In conclusion, we have evidenced a laser-induced spectral shift of single molecule lines close to a metallic surface. Its linear dependence on the excitation intensity and on the inverse of the dephasing rate is qualitatively explained by a simple theoretical model of a laser-driven molecule self-coupled by a mirror. Further developments

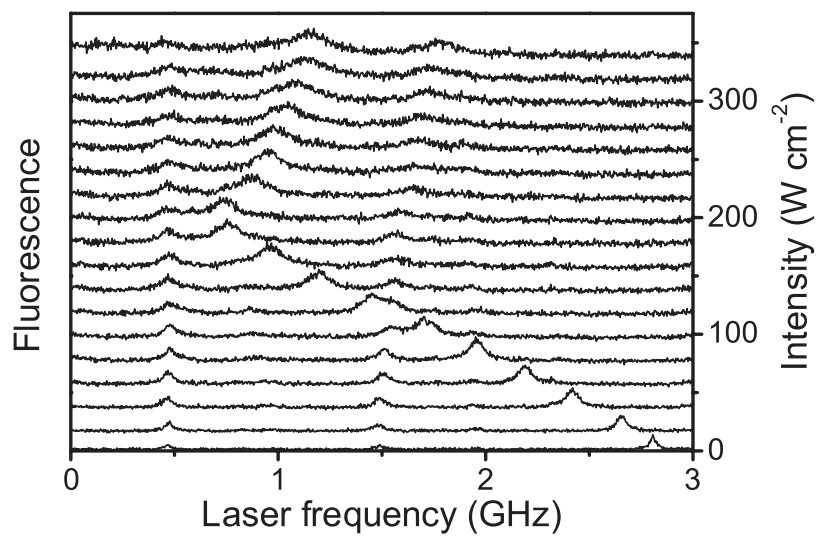

FIG. 4. Series of excitation spectra for several single molecules of DBATT in hexadecane near a $50 \mathrm{~nm}$ gold layer, at $T=$ $1.7 \mathrm{~K}$. The corresponding excitation intensity is reported on the right scale. The molecule presenting a large LIS effect (slope of $\left.11 \mathrm{MHz} /\left(\mathrm{W} \mathrm{cm}^{-2}\right)\right)$ undergoes a turning point in the shift at high excitation intensity (higher than $\sim 100 I_{\text {sat }}$ ). 
will be the control of the molecule-surface distance and orientation, as well as the elaboration of more sophisticated theoretical models to explain the LIS behavior in different experimental conditions such as metal nature and intensity regime.

We acknowledge C. Cohen Tannoudji and A. Bouzdine for helpful discussions in the early stage of the work. We thank C. Jay, T. Cohen-Bouhacina, and J. P. Aimé for AFM characterization of the metallic films, as well as J. Salardenne for his help in the preparation of the metallic layers. This work was supported by CNRS, ANR, Région Aquitaine, and the French Ministry for Education and Research (MENRT).

*Email address: b.lounis@cpmoh.u-bordeaux1.fr

[1] W. L. Barnes, J. Mod. Opt. 45, 661 (1998).

[2] P.W. Milonni, The Quantum Vacuum (Academic, New York, 1994).

[3] H. Morawitz, Phys. Rev. 187, 1792 (1969).

[4] G. S. Agarwal, Phys. Rev. A 11, 230 (1975).

[5] G. S. Agarwal, Phys. Rev. A 12, 1475 (1975).

[6] R. Chance, A. Prock, and R. Silbey, Adv. Chem. Phys. 37, 1 (1978).

[7] J. M. Wylie and J. E. Sipe, Phys. Rev. A 32, 2030 (1985).

[8] D. Meschede, W. Jhe, and E. A. Hinds, Phys. Rev. A 41, 1587 (1990).
[9] K. H. Drexhage, in Progress in Optics, edited by E. Wolf (North-Holland, Amsterdam, 1974), Vol. 12, p. 163.

[10] D. Bloch and M. Ducloy, in Advances In Atomic, Molecular, And Optical Physics, edited by B. Bederson and H. Walther (Academic, New York, 2005), Vol. 50, p. 91.

[11] M. A. Wilson et al., Phys. Rev. Lett. 91, 213602 (2003).

[12] P. Tamarat et al., J. Phys. Chem. A 104, 1 (2000).

[13] B. C. Buchler et al., Phys. Rev. Lett. 95, 063003 (2005).

[14] P. Anger, P. Bharadwaj, and L. Novotny, Phys. Rev. Lett. 96, 113002 (2006).

[15] S. Kuhn et al., Phys. Rev. Lett. 97, 017402 (2006).

[16] T. Basche et al., Single-Molecule Optical Detection, Imaging and Spectroscopy (VCH, Weinheim, Germany, 1997).

[17] B. Lounis, F. Jelezko, and M. Orrit, Phys. Rev. Lett. 78, 3673 (1997).

[18] L. Fleury et al., Chem. Phys. Lett. 236, 87 (1995).

[19] A. M. Boiron, B. Lounis, and M. Orrit, J. Chem. Phys. 105, 3969 (1996).

[20] H. Morawitz and M. R. Philpott, Phys. Rev. B 10, 4863 (1974).

[21] W. H. Weber and C. F. Eagen, Opt. Lett. 4, 236 (1979).

[22] G. S. Agarwal and S. Dutta Gupta, quant-phys/0011098.

[23] M. L. Philpott, J. Chem. Phys. 62, 1812 (1975).

[24] The surface plasmon resonance could be accounted for by including the dispersion of the parameter beta in Eq. (1). This can be done following, for example, the calculation given in Ref. [22]. Note that the dispersion of beta would be centered around the surface plasmon resonance. 